\title{
Dermatoses in the hospital and their impact on quality of life
}

\author{
Kaoutar Sof', Soraya Aouali', Sara Bensalem', Nada Zizi' ${ }^{1,2}$, Siham Dikhaye ${ }^{1,2}$
}

${ }^{1}$ Department of Dermatology, Mohammed 6 University Hospital of Oujda - Medical School of Oujda, Mohammed First University of Oujda, Morocco, ${ }^{2}$ Laboratory of Epidemiology, Clinical research and Public Health, Medical School of Oujda, Mohammed First University of Oujda, Morocco

Corresponding author: Kaoutar Sof, MD, E-mail: kaouthar.sof@gmail.com

Sir,

Dermatological pathologies may be responsible for the creation of a real handicap, affecting the patient's self-esteem and their professional and social life. The aim of this study was to assess the impact of diseases on the quality of life of patients hospitalized at the dermatology department.

The following was a retrospective study that included patients over eighteen years of age, hospitalized at the dermatology department of Hospital Mohammed VI in Oujda from January 2018 through December 2019. The Arabic version of the validated DLQI was used for all patients [1].

A total of 294 patients were collected, with a mean age of 53.95 years and a male-to-female ratio of 0.85 .

The most frequent reasons for hospitalization were infectious dermo-hypodermitis $(n=51)$, autoimmune bullous dermatosis $(n=23)$, severe drug eruption $(n=20)$, genodermatosis $(n=17)$, melanocytic $(n=9)$ and non-melanocytic skin tumors $(n=17)$, severe psoriasis $(n=17)$, cutaneous lymphoma $(n=11)$, alopecia areata $(n=10)$, dermatomyositis $(n=8)$, and Verneuil's disease $(n=5)$.

The DLQI was impossible to calculate in eleven patients. The mean DLQI in all patients was 10.20 , corresponding to a moderate effect on quality of life.

The mean DLQI was as follows: Verneuil's disease at 17.4, severe psoriasis at 16.6, dermatomyositis at
14.42 , genodermatosis at 12.37 , cutaneous lymphoma at 11.45 , severe drug eruption at 11 , alopecia areata at 10.5, AIBD at 9.67, skin tumors at 7.76, and infectious dermo-hypodermitis at 7.52 .

The DLQI was the first index measuring quality of life in dermatology and is still widely used today[2]. The number of publications concerning the impact of dermatological pathologies on quality of life has increased in recent years [3].

Our results showed that the DLQI was higher in patients with Verneuil's disease, severe psoriasis, and dermatomyositis.

These results agree with the data of the literature, many publications have shown that psoriasis seriously impaired the quality of life and was responsible of social anxiety in patients [4]. Verneuil's disease is also responsible of a significant impairment on quality of life mainly due to the sexual disorders caused by this pathology [5].

Another study on dermatomyositis showed that there is a significant correlation between the severity of skin signs and the quality of life of patients [6].

Dermatological pathologies are distinguished from other pathologies by their displaying character, which is responsible for a significant impact on the patient's quality of life. The management of dermatology patients requires psychological support in addition to conventional therapy. However, these pathologies are still not recognized as long-term illnesses in Morocco.

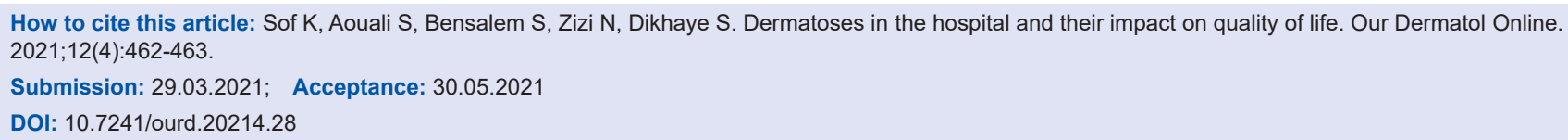




\section{Statement of Human and Animal Rights}

All the procedures followed were in accordance with the ethical standards of the responsible committee on human experimentation (institutional and national) and with the 2008 revision of the Declaration of Helsinki of 1975.

\section{Statement of Informed Consent}

Informed consent for participation in this study was obtained from all patients.

\section{REFERENCES}

1. Khoudri I. Traduction, adaptation transculturelle et validation de la version arabe pour le Maroc du Dermatology Life Quality Index (DLQI). Rev Épidémiol Santé Publiq. 2009;5:35.
2. Council ML. Quality of life and the dermatologist. JAMA Dermatology. 2018;6:639-744.

3. Chernyshov PV. The evolution of quality of life assessment and use in dermatology. Dermatology. 2019;235:167-74.

4. Yildirim FE, Şeremet S, Afşar FŞ, Yildiz İ, İyidoğan E. Evaluation of social anxiety levels and related factors in psoriasis patients: A controlled, cross-sectional study. Noro Psikiyatr Ars. 2020;57:148-53.

5. Cuenca-Barrales C, Montero-Vílchez T, Szepietowski JC, Matusiak L, Molina-Leyva A. Sexual impairment in patients with hidradenitis suppurativa: A systematic review. J Eur Acad Dermatol Venereol. 2021;35:345-52.

6. Goreshi R, Chock M, Foering K, Feng R, Okawa J, Rose M, Fiorentino D, Werth V. Quality of life in dermatomyositis. J Am Acad Dermatol. 2011;65:1107-6.

Copyright by Kaoutar Sof, et al. This is an open access article distributed under the terms of the Creative Commons Attribution License, which permits unrestricted use, distribution, and reproduction in any medium, provided the original author and source are credited.

Source of Support: Nil, Conflict of Interest: None declared. 